\title{
Aerodynamic forces and flow fields of a two-dimensional hovering wing
}

\author{
K. B. Lua $\cdot$ T. T. Lim $\cdot$ K. S. Yeo
}

Published online: 12 August 2008

(C) Springer-Verlag 2008

\section{Erratum to: Exp Fluids}

\section{DOI 10.1007/s00348-008-0527-z}

The original version of this article unfortunately contained some mistakes.

The composition and labelling of Figs. 6, 8, 12 and 13 have been mixed up.

The correct figures are given on the following pages.

The online version of the original article can be found under doi:10.1007/s00348-008-0527-z.

K. B. Lua $(\bowtie) \cdot$ T. T. Lim · K. S. Yeo Department of Mechanical Engineering, National University of Singapore,

10 Kent Ridge Crescent, Singapore, Singapore

e-mail: mpelkb@nus.edu.sg 

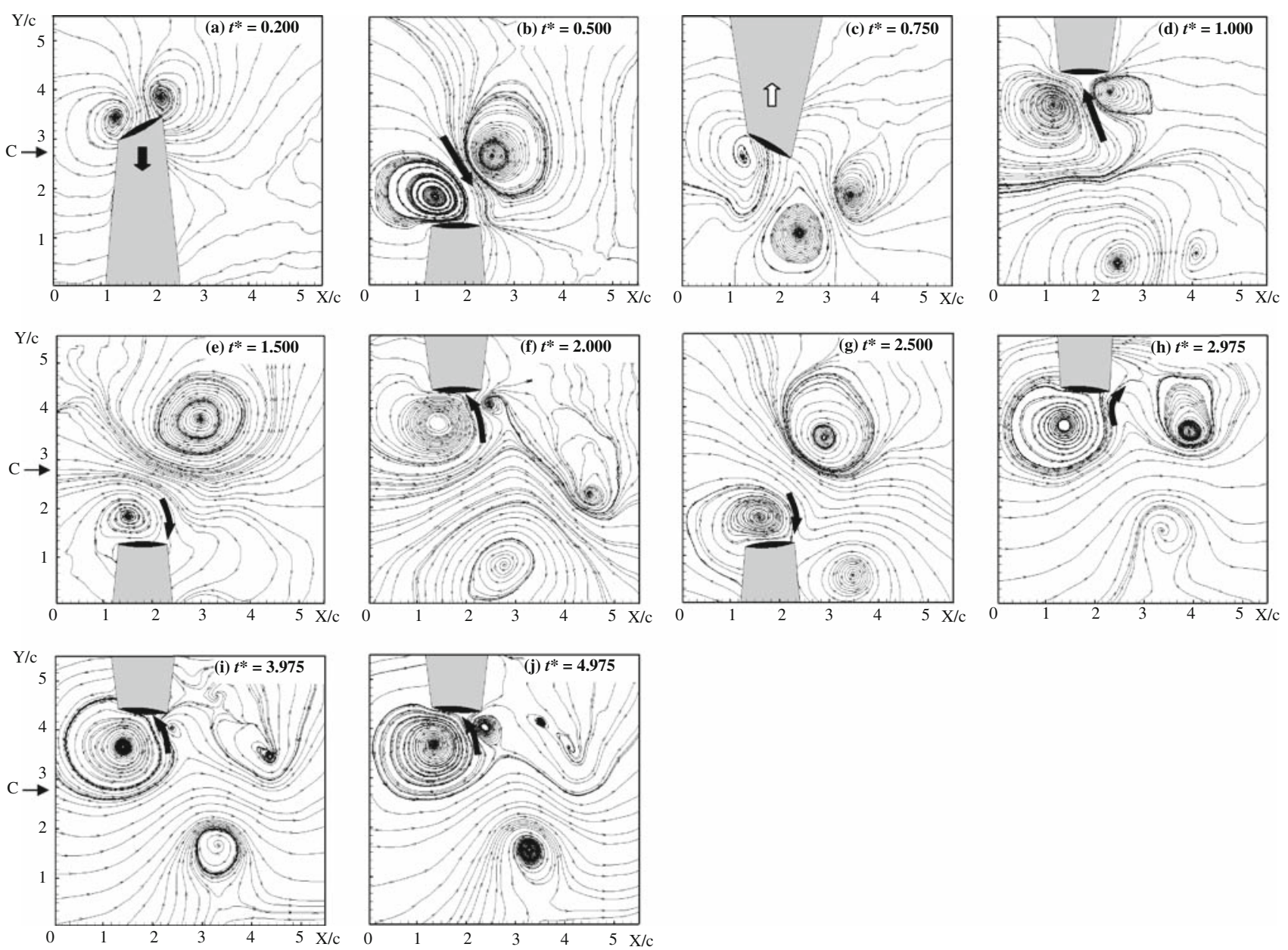

Fig. 6 The instantaneous streamline patterns for those instances indicated by the same labels in Fig. 5. " $C$ " 'indicates the center of the flapping motion 
Fig. 8 f-j Instantaneous streamline patterns (left column) and the corresponding vorticity fields (right column) during the upstroke of the fifth cycle of flapping for $\alpha_{\mathrm{A}}=30^{\circ}$. The length of the reference arrow indicates ten times the $U_{\text {rms }}$. Note that the time sequence is intentionally arranged from bottom to top of the figure to coincide with the direction of the upstroke
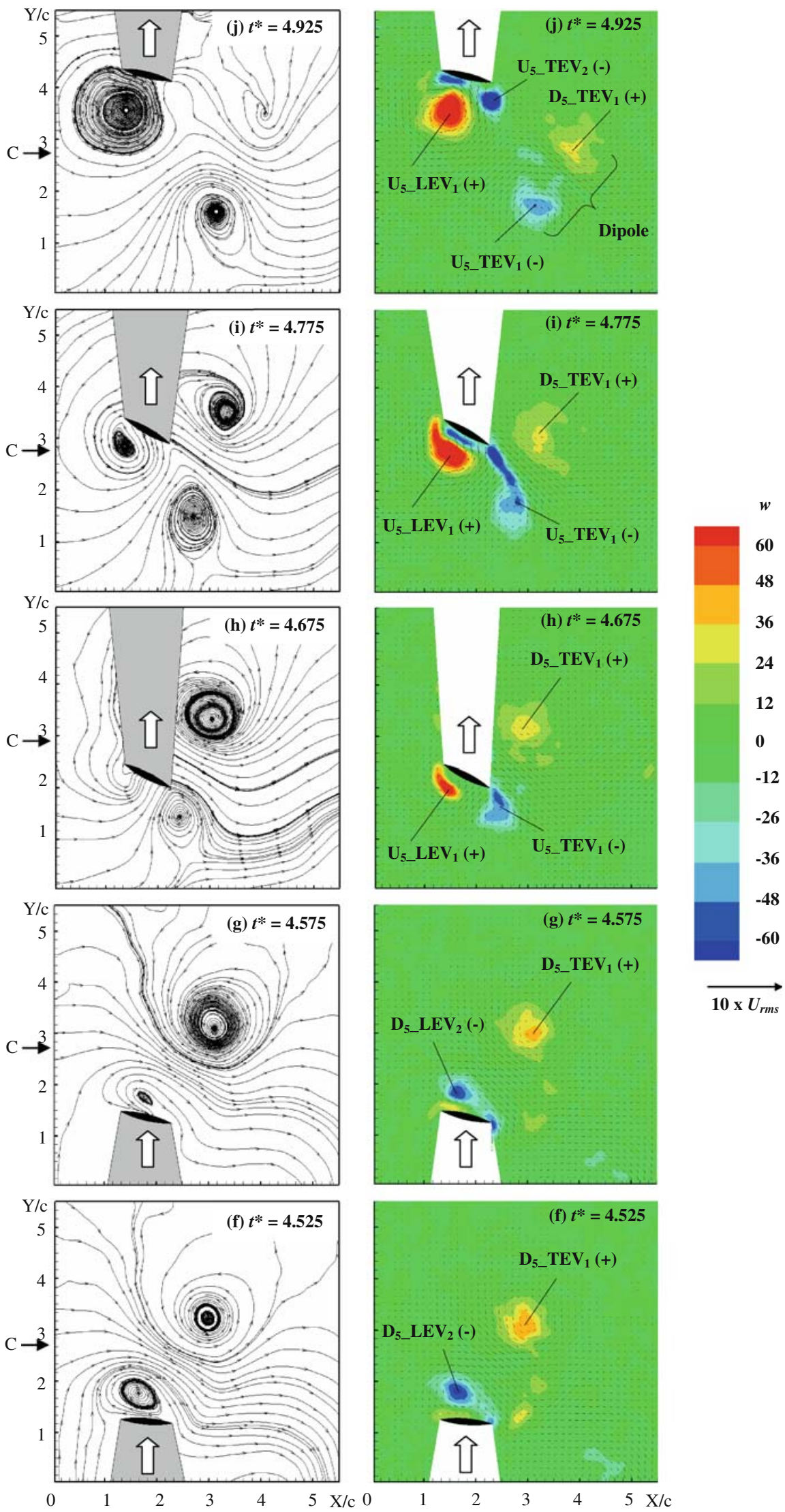
Fig. 12 e-h Instantaneous streamline patterns (left column) and the corresponding vorticity fields (right column) during the upstroke of the fifth cycle of flapping with $R e=663$ and $\alpha_{\mathrm{A}}=60^{\circ}$. " $C$ '" indicates the center of heaving motion. Note that the time sequence is intentionally arranged from bottom to top of the figure to coincide with the upstroke. The length of the reference arrow indicates ten times the $U_{\text {rms }}$
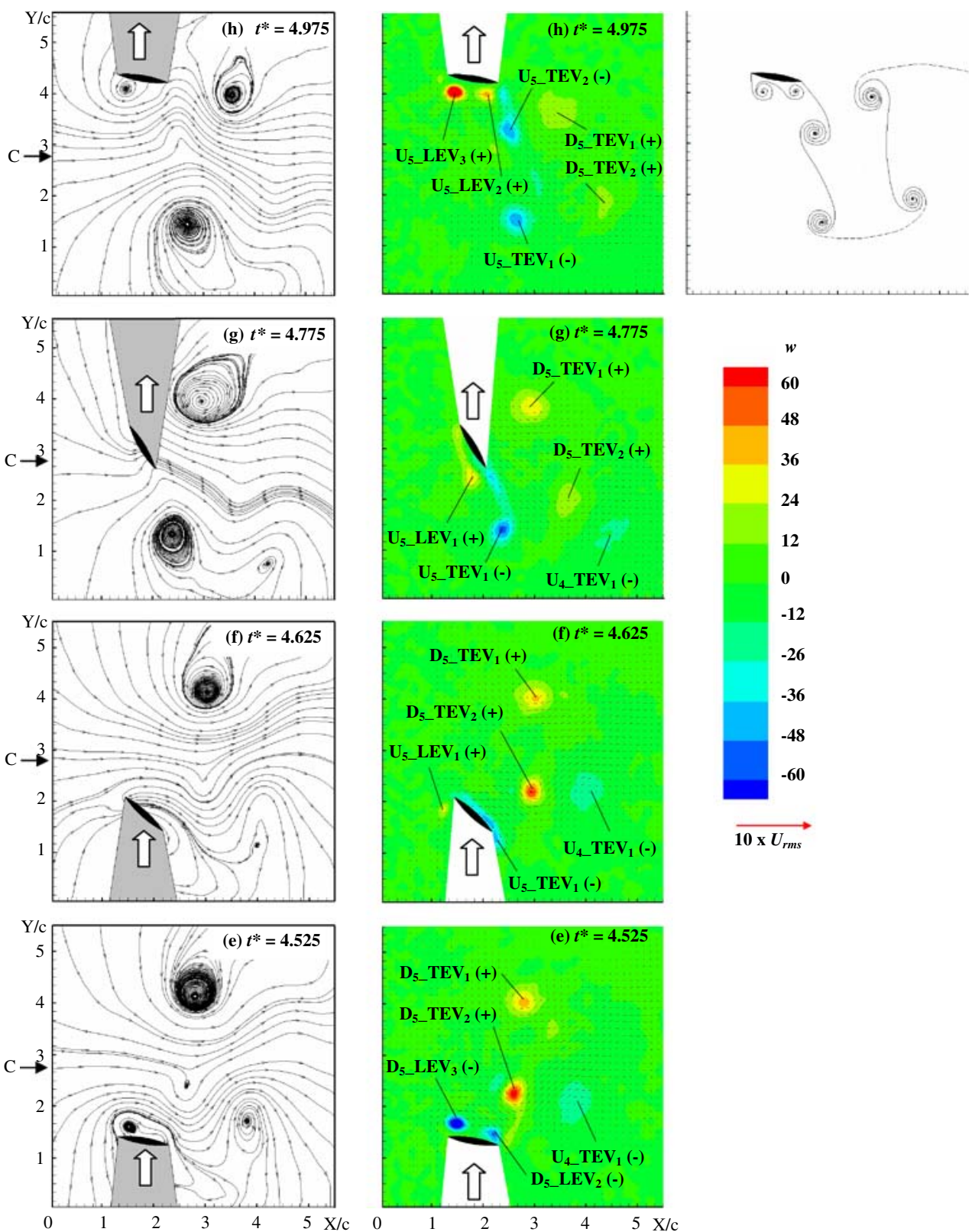
Fig. 13 Velocity and vorticity fields during the ninth cycle of flapping with $R e=2,652$ and $\alpha_{\mathrm{A}}=30^{\circ}$. a Downstroke.

b Upstroke. " $C$ '" indicates the center of heaving motion. The length of the reference arrow indicates ten times the $U_{\text {rms }}$
(A) Downstroke
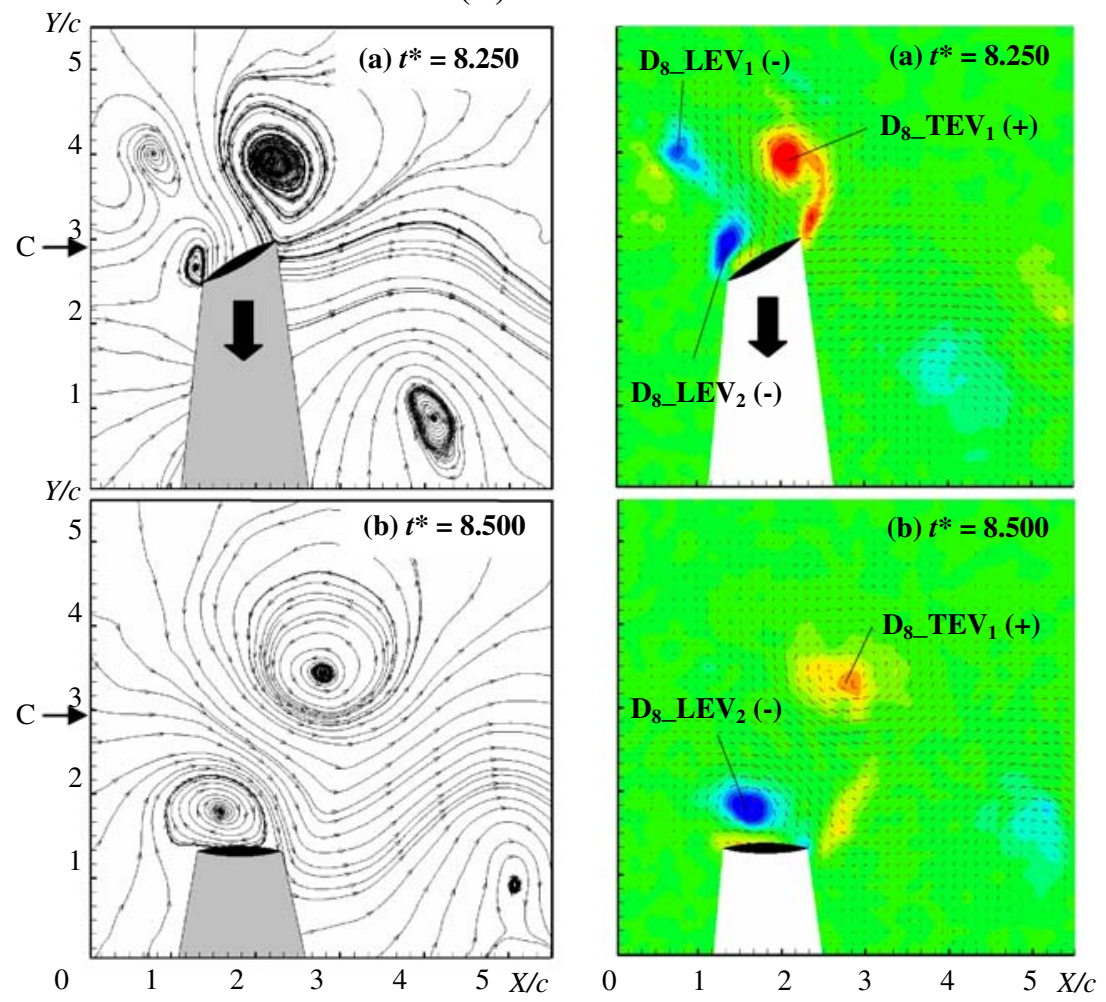

$w$

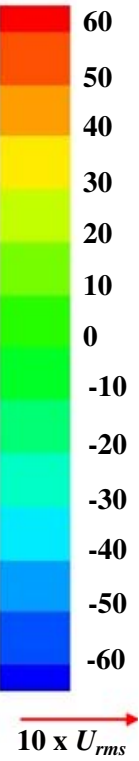

(B) Upstroke
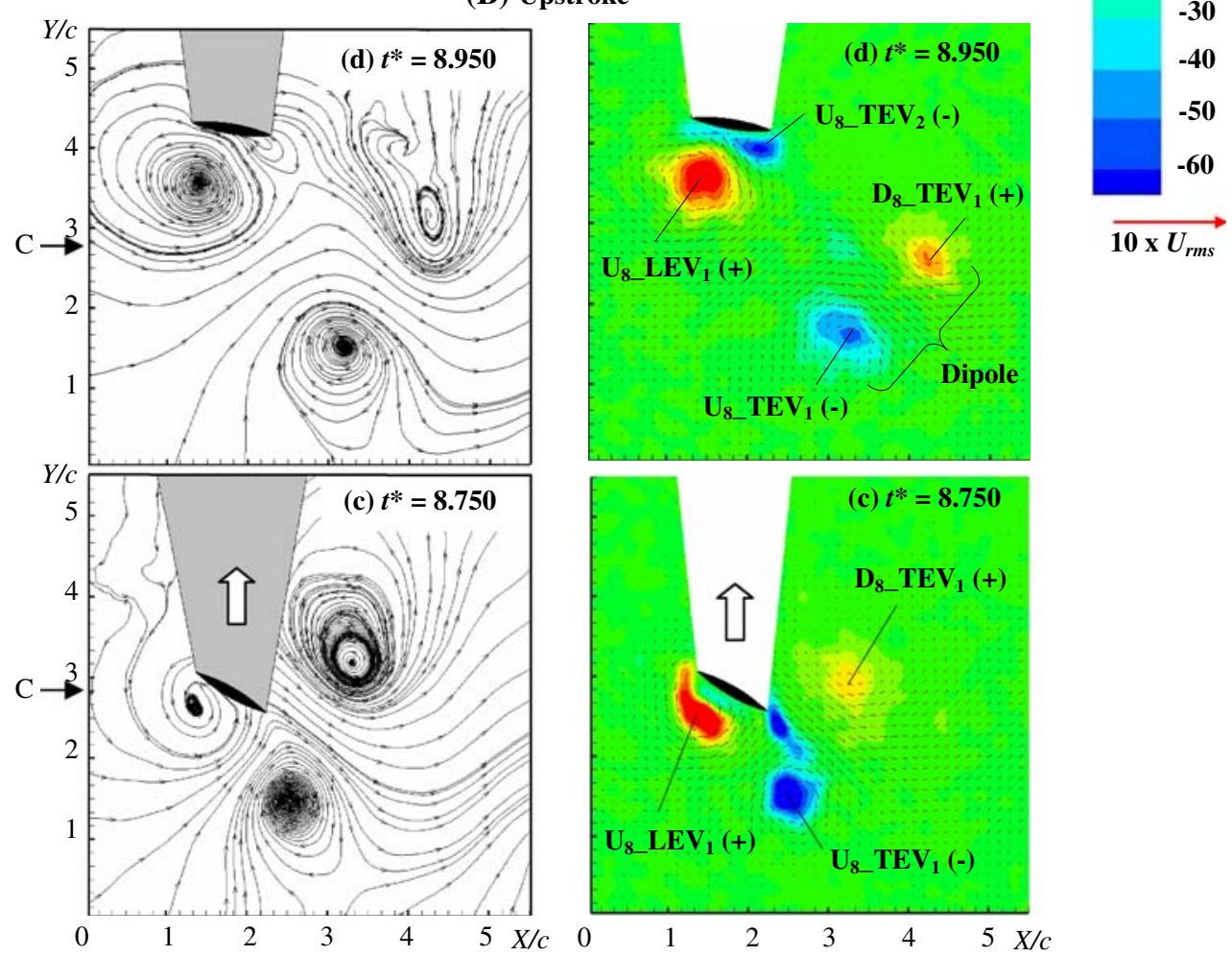